\title{
A Systematic Study of IRAS Selected Proto-Planetary Nebula Candidates III. The Northern Sources
}

\author{
Jingyao $\mathrm{Hu}$ \\ National Astronomical Observatories, Chinese Academy of Sciences \\ 20A Datun Road, Beijing 100012, China \\ hjy@bao.ac.cn
}

We have selected a PPN candidates sample from IRAS color-color diagram. The selected criteria, the objects list of whole sample and photometric and spectroscopic observational results for southern objects have been published (paper I, $\mathrm{Hu}$ et al, 1993), and $\mathrm{CO}$ and $\mathrm{OH}$ maser observations for the total sample are published in 1994 (paper II, Hu et al, 1994). The 20 northern objects were observed. The optical photometric observations were taken at the J. Kapteyn telescope with CCD camera, infrared photometric observations were obtained with 1.26 meter infrared telescope at Xinglong station, Beijing Observatory, and spectroscopic observational observations were carried out using the FOS attached on the 2.5 meter Issac Newton telescope in 1989; in 1996 and 2001 we re-observed these objects using $2.16 \mathrm{~m}$ telescope and OMR spectroscopy at Xinglong station, Beijing Observatory. We have not found any variations between two epochs for most objects in their optical spectra except IRAS $19367+2458$. The spectra we obtained in 1989 IRAS $19367+2458$ is a planetary nebula with [WC] core star (Hu and Dong, 1992), but in our spectra obtained in 1996 and 2001 it is a M-type star. There are two possibilities: 1. We made mistakes of identification, if so, where is the planetary nebula? 2. It varies, but we can not find any difference in the spectra obtained in 1996 and 2001. The question is still open, more observations are needed in the future.

A paper on the observational results and discussions for all northern objects will submitted to the Chinese Journal of Astronomy and Astrophysics.

\section{References}

Hu J.Y. and Dong Y.S., 1992 Chinese Science Bulletin, 37, 213

Hu J.Y., Slijkhuis S., de Jong T., Jiang B.W. 1993, A\&AS 100, 413 (paper I)

Hu J.Y., te Lintel Hekkert P., Slijkhuis S., Baas F. 1994, A\&AS 103, 301 (paper II) 